\title{
Perturbation calculation of the axial anomaly of a Ginsparg-Wilson lattice Dirac operator
}

\author{
Ting-Wai Chiu* and Tung-Han Hsieh \\ Department of Physics, National Taiwan University, Taipei, Taiwan 106, Taiwan
}

(Received 21 September 2001; revised manuscript received 7 November 2001; published 15 February 2002)

\begin{abstract}
A recent proposal suggests that even if a Ginsparg-Wilson lattice Dirac operator does not possess any topological zero modes in topologically nontrivial gauge backgrounds, it can reproduce the correct axial anomaly for sufficiently smooth gauge configurations, provided that it is exponentially local, doubler-free, and has the correct continuum behavior. In this paper, we calculate the axial anomaly of this lattice Dirac operator in weak coupling perturbation theory, and show that it recovers the topological charge density in the continuum limit.
\end{abstract}

DOI: 10.1103/PhysRevD.65.054508

\section{INTRODUCTION}

Recently, one of us (T.W.C.) has constructed [1] a Ginsparg-Wilson Dirac operator that is $\gamma_{5}$ Hermitian, exponentially local, doubler-free, and has the correct continuum behavior, but it does not possess any topological zero modes for topologically nontrivial background gauge fields. This suggests that one might have the option to turn off the topological zero modes of a Ginsparg-Wilson lattice Dirac operator, without affecting its physical behavior (axial anomaly, fermion propagator, etc.) at least for the topologically trivial gauge sector. Therefore it is interesting to verify explicitly that it indeed reproduces the continuum axial anomaly for smooth gauge backgrounds, in the framework of weak coupling perturbation theory which is amenable to analytic calculations.

The Ginsparg-Wilson lattice Dirac operator proposed in Ref. [1] is

$$
D=a^{-1} D_{c}\left(1+r D_{c}\right)^{-1}, \quad r>0,
$$

with

$$
\begin{aligned}
D_{c} & =\sum_{\mu} \gamma^{\mu} T^{\mu}, \quad T^{\mu}=f t^{\mu} f, \\
f & =\left(\frac{2 c}{\sqrt{t^{2}+w^{2}}+w}\right)^{1 / 2}, \quad t^{2}=-\sum_{\mu} t^{\mu} t^{\mu} .
\end{aligned}
$$

Here $\gamma^{\mu} t^{\mu}$ is the naive lattice fermion operator and $-w$ is the Wilson term with a negative mass $-c(0<c<2)$ :

$$
\begin{aligned}
t^{\mu}(x, y) & =\frac{1}{2}\left[U_{\mu}(x) \delta_{x+\hat{\mu}, y}-U_{\mu}^{\dagger}(y) \delta_{x-\hat{\mu}, y}\right], \\
U_{\mu}(x) & =\exp \left[i a g A_{\mu}\left(x+\frac{a}{2} \hat{\mu}\right)\right], \\
\gamma^{\mu} & =\left(\begin{array}{cc}
0 & \sigma_{\mu} \\
\sigma_{\mu}^{\dagger} & 0
\end{array}\right),
\end{aligned}
$$

*Email address: twchiu@phys.ntu.edu.tw
PACS number(s): 11.15.Ha, 11.30.Fs, 11.30.Rd

$$
\begin{aligned}
\sigma_{\mu} \sigma_{\nu}^{\dagger}+\sigma_{\nu} \sigma_{\mu}^{\dagger}= & 2 \delta_{\mu \nu}, \\
w(x, y)= & c-\frac{1}{2} \sum_{\mu}\left[2 \delta_{x, y}-U_{\mu}(x) \delta_{x+\hat{\mu}, y}\right. \\
& \left.-U_{\mu}^{\dagger}(y) \delta_{x-\hat{\mu}, y}\right], \quad 0<c<2,
\end{aligned}
$$

where the Dirac, color, and flavor indices have been suppressed. Note that in Eq. (1), we do not fix $r=1 / 2 c$, but keep it as a free parameter, since we intend to show that the axial anomaly is independent of $r$ in the continuum limit.

Evidently, the lattice Dirac operator (1) is $\gamma_{5}$ Hermitian,

$$
D^{\dagger}=\gamma_{5} D \gamma_{5},
$$

and satisfies the Ginsparg-Wilson relation [2]

$$
D \gamma_{5}+\gamma_{5} D=2 \operatorname{raD} \gamma_{5} D .
$$

In the free fermion limit, $D$ is exponentially-local, doublerfree, and has correct continuum behavior [1]. These properties should be sufficient to guarantee that $D$ can reproduce the correct axial anomaly in a smooth gauge background.

In the next section, we calculate the axial anomaly of Eq. (1) in weak coupling perturbation theory, and show that, in the continuum limit, it recovers the topological charge density of the gauge background

$$
\frac{g^{2}}{32 \pi^{2}} \sum_{\mu \nu \lambda \sigma} \epsilon_{\mu \nu \lambda \sigma} \operatorname{tr}\left(F_{\mu \nu} F_{\lambda \sigma}\right),
$$

where

$$
F_{\mu \nu}=\partial_{\mu} A_{\nu}-\partial_{\nu} A_{\mu}+i g\left[A_{\mu}, A_{\nu}\right] .
$$

Before we proceed to the perturbation calculations in the next section, we first clarify that even though a GinspargWilson lattice Dirac operator can reproduce the continuum axial anomaly for sufficiently smooth gauge configurations, it does not necessarily imply that it could possess topological zero modes for topologically nontrivial gauge backgrounds. This can be seen as follows.

Consider a gauge configuration with positive definite topological charge $Q>0$, and its topological charge density $\rho(x)$ is very smooth for all $x$, 


$$
\int d^{4} x \rho(x)=Q
$$

Then, in principle, $\rho(x)$ can be decomposed into two parts,

$$
\rho(x)=\rho_{w}(x)+\rho_{0}(x),
$$

where $\rho_{w}(x)$ is the "winding number density," which is positive for all $x$, and it is the smoothest particular solution of Eq. (11),

$$
\int d^{4} x \rho_{w}(x)=Q, \quad \rho_{w}(x)>0 \quad \forall x,
$$

and $\rho_{0}(x)$ is the solution of the homogeneous equation (11),

$$
\int d^{4} x \rho_{0}(x)=0
$$

If one assumes that the amount of the topological charge $Q$ is bounded by a constant times the square root of the spacetime volume, then in the infinite volume and continuum limit, $\rho_{w}(x)$ becomes infinitesimally small at any $x$; thus it becomes unobservable. In other words, even if one calculates the axial anomaly $\mathcal{A}(x)$ [Eq. (15)] of a Ginsparg-Wilson lattice Dirac operator, and shows that it agrees with $\rho(x)$ $\simeq \rho_{0}(x)$ in the infinite volume and continuum limit; one still cannot be sure whether the integral $\int d^{4} x \mathcal{A}(x)$ is equal to the topological charge $Q$ or not.

In order to have a definite answer to this question, one needs to perform a nonperturbative calculation of the axial anomaly on a finite lattice. Otherwise, the "winding number density" $\rho_{w}(x)$ could hardly be detected at a site. However, to our knowledge, there is no previous nonperturbative analytic calculations of the axial anomaly for any GinspargWilson lattice Dirac operator on a finite lattice. Therefore, in this paper, we do not intend to tackle the difficult problem of performing a nonperturbative analytic calculation of the axial anomaly on a finite lattice.

Nevertheless, from the argument presented in Ref. [1], it is clear that Eq. (1) does not possess any topological zero modes. Thus, it follows that, on a finite lattice, the axial anomaly for at least some of the lattice sites is not in agreement with the topological charge density, since its sum over all sites is equal to the zero index, rather than the nonzero topological charge $Q$ of the gauge background. Therefore, for any topologically nontrivial gauge background, we have no difficulties to understand why the axial anomaly of Eq. (1) cannot be exactly equal to the topological charge density for all sites on a finite lattice. However, in the infinite volume and continuum limit, $\rho_{w}(x) \rightarrow 0$, then the axial anomaly $\mathcal{A}(x)$ can appear to be in agreement with the topological charge density $\rho(x)$ for sufficiently smooth gauge backgrounds, even though the integral $\int d^{4} x \mathcal{A}(x)$ is always zero. Thus, for a sufficiently smooth gauge background (except for any possible "winding" at the infinity), we can calculate the axial anomaly (at any point except the infinity) of the lattice Dirac operator (1) in weak coupling perturbation theory, and show that in the continuum limit, it agrees with the topological charge density of the gauge background, regardless of the global topological charge.

\section{PERTURBATION CALCULATION OF THE AXIAL ANOMALY}

The axial anomaly ${ }^{1}$ of a flavor-singlet of Ginsparg-Wilson lattice fermions can be written as [3]

$$
\begin{aligned}
\mathcal{A}(x) & =\operatorname{tr}\left[\gamma_{5}(1-r a D)(x, x)\right] \\
& =\int \frac{d^{4} p}{(2 \pi)^{4}} \int \frac{d^{4} q}{(2 \pi)^{4}} e^{i(p-q) \cdot x} \operatorname{tr}\left[\gamma_{5}(1-r a D)(p, q)\right],
\end{aligned}
$$

where the trace runs over the Dirac, color, and flavor space. Now we expand $D$ in power series of the gauge coupling (through the link variables)

$$
D=D_{0}+D_{1}+D_{2}+D_{3}+D_{4}+O\left(g^{5}\right),
$$

where $D_{n}$ denotes the terms containing the factor $g^{n}$, the gauge coupling to the $n$th power. Then we have

$$
\begin{aligned}
1-r a D= & e_{0}-e_{0} d_{1} e_{0}-e_{0}\left(d_{2}-d_{1} e_{0} d_{1}\right) e_{0} \\
& -e_{0}\left(d_{3}-d_{1} e_{0} d_{2}-d_{2} e_{0} d_{1}+d_{1} e_{0} d_{1} e_{0} d_{1}\right) e_{0} \\
& -e_{0}\left(d_{4}-d_{1} e_{0} d_{3}-d_{3} e_{0} d_{1}-d_{2} e_{0} d_{2}+d_{1} e_{0} d_{1} e_{0} d_{2}\right. \\
& \left.+d_{1} e_{0} d_{2} e_{0} d_{1}+d_{2} e_{0} d_{1} e_{0} d_{1}-d_{1} e_{0} d_{1} e_{0} d_{1} e_{0} d_{1}\right) e_{0} \\
& +O\left(g^{5}\right)
\end{aligned}
$$

where

$$
\begin{aligned}
e_{0}= & 1-r a D_{0} \equiv\left(1+d_{0}\right)^{-1} \\
d_{1}= & r \sum_{\mu} \gamma_{\mu}\left(f_{1} t_{0}^{\mu} f_{0}+f_{0} t_{1}^{\mu} f_{0}+f_{0} t_{0}^{\mu} f_{1}\right) \\
d_{2}= & r \sum_{\mu} \gamma_{\mu}\left(f_{2} t_{0}^{\mu} f_{0}+f_{0} t_{2}^{\mu} f_{0}+f_{0} t_{0}^{\mu} f_{2}+f_{1} t_{1}^{\mu} f_{0}+f_{1} t_{0}^{\mu} f_{1}\right. \\
& \left.+f_{0} t_{1}^{\mu} f_{1}\right) \\
d_{3}= & r \sum_{\mu} \gamma_{\mu} \sum_{i=0}^{3} \sum_{j=0}^{3-i}\left(f_{i} t_{j}^{\mu} f_{3-i-j}\right) \\
d_{4}= & r \sum_{\mu} \gamma_{\mu} \sum_{i=0}^{4} \sum_{j=0}^{4-i}\left(f_{i} t_{j}^{\mu} f_{4-i-j}\right) \\
f= & f_{0}+f_{1}+f_{2}+f_{3}+f_{4}+O\left(g^{5}\right)
\end{aligned}
$$

\footnotetext{
${ }^{1}$ It is understood that the axial anomaly (15) at a lattice site has to be normalized by the volume of the unit cell, $a^{4}$, when it is compared with the topological charge density in the continuum.
} 


$$
t^{\mu}=t_{0}^{\mu}+t_{1}^{\mu}+t_{2}^{\mu}+t_{3}^{\mu}+t_{4}^{\mu}+O\left(g^{5}\right) .
$$

Evidently some terms in Eq. (17) do not contribute to the axial anomaly, since they only have a factor of a product less than four distinct $\gamma$ matrices; thus they vanish after taking the trace with $\gamma_{5}$ in Eq. (15). Note that each term of $d_{n}$ only has one factor of $\gamma$ matrix, and $e_{0}$ contributes at most one factor of $\gamma$ matrix [see Eq. (33)]. Thus, the terms $e_{0}$ and $e_{0} d_{n} e_{0}$ (for any $n$ ) can be dropped from the series, when Eq. (17) is substituted into Eq. (15).

Then the axial anomaly at the order $g^{2}$ is due to the fourth term in Eq. (17),

$$
\begin{aligned}
\mathcal{A}_{2}(x)= & \int_{p} \int_{q} \int_{k} e^{i(p-q) \cdot x} \\
& \times \operatorname{tr}\left[\gamma_{5} e_{0}(p) d_{1}(p, k) e_{0}(k) d_{1}(k, q) e_{0}(q)\right],
\end{aligned}
$$

where

$$
\int_{p} \equiv \int \frac{d^{4} p}{(2 \pi)^{4}},
$$

etc.

Similarly, substituting higher-order terms of Eq. (17) into Eq. (15) gives the axial anomaly at orders $g^{3}$ and $g^{4}$. Although there seems to be many terms involving higher-order vertices $\left(d_{2}, d_{3}\right.$, etc. $)$ at higher orders, the nonvanishing contributions at orders $g^{3}$ and $g^{4}$ only come from terms $e_{0} d_{1} e_{0} d_{1} e_{0} d_{1} e_{0}$ and $e_{0} d_{1} e_{0} d_{1} e_{0} d_{1} e_{0} d_{1} e_{0}$, respectively, as we shall show later. At this point, it is instructive to identify $e_{0}=\left(1+d_{0}\right)^{-1}[$ Eq. (18)] with the free fermion propagator, $d_{1}$ [Eq. (19)] with the "quark-quark-gluon" vertex, $d_{2}$ [Eq. (20)] with the "quark-quark-gluon-gluon" vertex, etc. Then the expression $\operatorname{tr}\left(\gamma_{5} e_{0} d_{1} e_{0} d_{1} e_{0}\right)$ contributing to the axial anomaly at $O\left(g^{2}\right)$ [Eq. (25)] corresponds to the triangle diagram coupling the axial current to two external gauge bosons through an internal fermion loop. Similarly, the expressions $\operatorname{tr}\left(\gamma_{5} e_{0} d_{1} e_{0} d_{1} e_{0} d_{1} e_{0}\right)$ and $\operatorname{tr}\left(\gamma_{5} e_{0} d_{1} e_{0} d_{1} e_{0} d_{1} e_{0} d_{1} e_{0}\right)$, contributing to the axial anomaly at $O\left(g^{3}\right)$ and $O\left(g^{4}\right)$, correspond to the quadrilateral (box) and the pentagon diagrams coupling the axial current to three and four external gauge bosons, respectively. These are exactly the Feynman diagrams $^{2}$ that contribute to the axial anomaly in continuum non-Abelian gauge theories [5]. Note that even though higher-order vertices are allowed at finite lattice spacing, symmetry forbids their contributions to the axial anomaly, thus only the "quark-quark-gluon" vertex $d_{1}$ can enter the fermion loop contributing to the axial anomaly. In the following three subsections, we evaluate the axial anomaly at orders $g^{2}, g^{3}$, and $g^{4}$ respectively.

\section{A. The axial anomaly at order $g^{2}$}

To evaluate Eq. (25), first we obtain $d_{1}$ [Eq. (19)] by expanding $f$ and $t^{\mu}$ in power series of the gauge coupling, Eqs. (23) and (24). Consider

$$
h=\sqrt{t^{2}+w^{2}}+w=h_{0}+h_{1}+h_{2}+h_{3}+h_{4}+O\left(g^{5}\right),
$$

which satisfies the identity $f \cdot f \cdot h=2 c$, i.e.,

$$
\left(f_{0}+f_{1}+\cdots\right)\left(f_{0}+f_{1}+\cdots\right)\left(h_{0}+h_{1}+\cdots\right)=2 c .
$$

To order $g$, it gives

$$
f_{0} f_{0} h_{0}=2 c,
$$

$$
f_{1} f_{0} h_{0}+f_{0} f_{1} h_{0}+f_{0} f_{0} h_{1}=0 \text {. }
$$

Solving these two equations, we obtain

$$
\begin{aligned}
f_{0}(p, k) & =\left(\frac{2 c}{\sqrt{t_{0}^{2}(p)+w_{0}^{2}(p)}+w_{0}(p)}\right)^{1 / 2} \delta^{4}(p-k) \\
& \equiv f_{0}(p) \delta^{4}(p-k), \\
f_{1}(p, k) & =-\frac{1}{2 c} \frac{f_{0}^{2}(p) f_{0}^{2}(k)}{f_{0}(p)+f_{0}(k)} h_{1}(p, k),
\end{aligned}
$$

where

$$
t_{0}^{2}(p)=-\sum_{\mu} t_{0}^{\mu}(p) t_{0}^{\mu}(p)=\sum_{\mu} \sin ^{2}\left(p_{\mu} a\right)
$$

Similarly, we obtain

$$
h_{1}(p, k)=\frac{w_{1}(p, k)\left[w_{0}(p)+w_{0}(k)\right]-\sum_{\mu} t_{1}^{\mu}(p, k)\left[t_{0}^{\mu}(p)+t_{0}^{\mu}(k)\right]}{\sqrt{t_{0}^{2}(p)+w_{0}^{2}(p)}+\sqrt{t_{0}^{2}(k)+w_{0}^{2}(k)}}+w_{1}(p, k) .
$$

Here we recall some well-known basic formulas in weak where coupling perturbation theory (see the Appendix):

$$
\begin{aligned}
& t^{\mu}(p, k)=t_{0}^{\mu}(p) \delta^{4}(p-k)+t_{1}^{\mu}(p, k)+O\left(g^{2}\right), \\
& w(p, k)=w_{0}(p) \delta^{4}(p-k)+w_{1}(p, k)+O\left(g^{2}\right),
\end{aligned}
$$

${ }^{2}$ See, for example, Fig. 22.2 in Ref. [4]. 


$$
\begin{aligned}
& t_{0}^{\mu}(p)=i \sin \left(p_{\mu} a\right) \\
& w_{0}(p)=c-\sum_{\mu}\left[1-\cos \left(p_{\mu} a\right)\right] \\
& t_{1}^{\mu}(p, k)=g \widetilde{A}_{\mu}(p-k) \partial_{\mu} t_{0}^{\mu}\left(\frac{p+k}{2}\right) \\
& w_{1}(p, k)=\sum_{\mu} g \widetilde{A}_{\mu}(p-k) \partial_{\mu} w_{0}\left(\frac{p+k}{2}\right), \\
& \widetilde{A}_{\mu}(p-k)=\sum_{x} e^{-i(p-k) \cdot[x+(a / 2) \hat{\mu}]} A_{\mu}\left(x+\frac{a}{2} \hat{\mu}\right) . \\
& \mathcal{A}_{2}(x)=g^{2} \int_{p} \int_{q} e^{i(p-q) \cdot x} \sum_{\mu, \nu} \int_{k} \operatorname{tr}\left\{\widetilde{A}_{\mu}(p-k) \tilde{A}_{\nu}(k-q)\right\} \\
& \times \operatorname{tr}\left[\gamma_{5} e_{0}(p) d_{1, \mu}(p, k) e_{0}(k) d_{1, \nu}(k, q) e_{0}(q)\right], \\
& e_{0}(p)=1-r a D_{0}(p)=\frac{1}{1+d_{0}(p)}=\frac{1-r f_{0}^{2}(p) \gamma \cdot t_{0}(p)}{1+r^{2} f_{0}^{4}(p) t_{0}^{2}(p)} \equiv b(p)+\sum_{\mu} \gamma^{\mu} c^{\mu}(p), \\
& d_{0}(p)=r f_{0}^{2}(p) \gamma \cdot t_{0}(p) \equiv \sum_{\mu} \gamma^{\mu} d_{0}^{\mu}(p), \\
& d_{1}(p, k)=g \sum_{\mu} \widetilde{A}_{\mu}(p-k) d_{1, \mu}(p, k), \\
& d_{1, \mu}(p, k)=r\left[f_{1, \mu}(p, k) \gamma \cdot t_{0}(k) f_{0}(k)+f_{0}(p) \gamma \cdot t_{0}(p) f_{1, \mu}(p, k)+f_{0}(p) \gamma^{\mu} \partial^{\mu} t_{0}^{\mu}\left(\frac{p+k}{2}\right) f_{0}(k)\right], \\
& f_{1, \mu}(p, k)=-\frac{1}{2 c} \frac{f_{0}^{2}(p) f_{0}^{2}(k)}{f_{0}(p)+f_{0}(k)}\left[\partial_{\mu} w_{0}\left(\frac{p+k}{2}\right)+\frac{\partial_{\mu} w_{0}((p+k) / 2)\left[w_{0}(p)+w_{0}(k)\right]-\partial_{\mu} t_{0}^{\mu}((p+k) / 2)\left[t_{0}^{\mu}(p)+t_{0}^{\mu}(k)\right]}{\sqrt{t_{0}^{2}(p)+w_{0}^{2}(p)}+\sqrt{t_{0}^{2}(k)+w_{0}^{2}(k)}}\right] .
\end{aligned}
$$

In the limit $k=p$, Eqs. (37) and (36) reduce to

$$
\begin{aligned}
& f_{1, \mu}(p, p)=\partial_{\mu} f_{0}(p), \\
& d_{1, \mu}(p, p)=\partial_{\mu}\left[r f_{0}(p) \gamma \cdot t_{0}(p) f_{0}(p)\right]=\partial_{\mu} d_{0}(p) .
\end{aligned}
$$

To evaluate the integral in Eq. (32), we change the variables $p \rightarrow p+k$ and $q \rightarrow q+k$; then Eq. (32) becomes

$$
\mathcal{A}_{2}(x)=g^{2} \int_{p} \int_{q} e^{i(p-q) \cdot x} \sum_{\mu, \nu} \operatorname{tr}\left\{\widetilde{A}_{\mu}(p) \widetilde{A}_{\nu}(-q)\right\} G_{\mu \nu}(p, q),
$$

where

$$
\begin{aligned}
G_{\mu \nu}(p, q)= & \int_{k} \operatorname{tr}\left[\gamma_{5} e_{0}(p+k) d_{1, \mu}(p+k, k) e_{0}(k)\right. \\
& \left.\times d_{1, \nu}(k, q+k) e_{0}(q+k)\right]
\end{aligned}
$$

In the following, we show that

$$
\begin{aligned}
G_{\mu \nu}(p, q) & =\left.\sum_{\lambda, \sigma} p_{\lambda} q_{\sigma} \frac{\partial}{\partial p_{\lambda}} \frac{\partial}{\partial q_{\sigma}} G_{\mu \nu}(p, q)\right|_{p, q=0}+O(a) \\
& =-M(c) \sum_{\lambda, \sigma} 4 p_{\lambda} q_{\sigma} \epsilon_{\mu \nu \lambda \sigma}+O(a)
\end{aligned}
$$

which, when substituted into Eq. (40), leads to the axial anomaly at the order $g^{2}$,

$$
\begin{aligned}
\mathcal{A}_{2}(x)= & g^{2} M(c) \sum_{\mu \nu \lambda \sigma} \epsilon_{\mu \nu \lambda \sigma} \operatorname{tr}\left\{\left(\partial_{\mu} A_{\nu}-\partial_{\nu} A_{\mu}\right)\right. \\
& \left.\times\left(\partial_{\lambda} A_{\sigma}-\partial_{\sigma} A_{\lambda}\right)(x)\right\}
\end{aligned}
$$

where $M(c)$ is a coefficient that tends to $1 / 32 \pi^{2}$ (for $0<c$ $<2$ ) in the continuum limit.

We expand $G_{\mu \nu}(p, q)$ in power series of $p$ and $q$, 


$$
\begin{aligned}
G_{\mu \nu}(p, q)= & G_{\mu \nu}(0,0)+\left.\sum_{\lambda} p_{\lambda} \frac{\partial}{\partial p_{\lambda}} G_{\mu \nu}(p, q)\right|_{p, q=0} \\
& +\left.\sum_{\sigma} q_{\sigma} \frac{\partial}{\partial q_{\sigma}} G_{\mu \nu}(p, q)\right|_{p, q=0} \\
& +\left.\sum_{\lambda, \sigma} p_{\lambda} q_{\sigma} \frac{\partial}{\partial p_{\lambda}} \frac{\partial}{\partial q_{\sigma}} G_{\mu \nu}(p, q)\right|_{p, q=0}+O(a) .
\end{aligned}
$$

It is easy to see that the zeroth order and the first-order terms in Eq. (43) vanish, by symmetry and the basic properties of $\gamma$ matrices,

$$
\begin{aligned}
\operatorname{tr}\left(\gamma_{5}\right) & =\operatorname{tr}\left(\gamma_{5} \gamma^{\alpha}\right) \\
& =\operatorname{tr}\left(\gamma_{5} \gamma^{\alpha} \gamma^{\beta}\right) \\
& =\operatorname{tr}\left(\gamma_{5} \gamma^{\alpha} \gamma^{\beta} \gamma^{\sigma}\right) \\
& =\operatorname{tr}\left(\gamma_{5} \gamma^{\alpha} \gamma^{\beta} \gamma^{\lambda} \gamma^{\sigma} \gamma^{\delta}\right)=0,
\end{aligned}
$$

$$
\operatorname{tr}\left(\gamma_{5} \gamma^{\alpha} \gamma^{\beta} \gamma^{\lambda} \gamma^{\sigma}\right)=\operatorname{tr}(\mathbb{1}) \epsilon_{\alpha \beta \lambda \sigma}
$$

Explicitly,

$$
\begin{aligned}
G_{\mu \nu}(0,0) & =\int_{k} \operatorname{tr}\left\{\gamma_{5} e_{0}(k) \partial_{\mu} d_{0}(k) e_{0}(k) \partial_{\nu} d_{0}(k) e_{0}(k)\right\} \\
& =\operatorname{tr}(\mathbb{1}) \int_{k} \epsilon_{\alpha \beta \delta \sigma} c^{\alpha}(k) c^{\delta}(k) b(k) \partial_{\mu} d_{0}^{\beta}(k) \partial_{\nu} d_{0}^{\sigma}(k),
\end{aligned}
$$

where Eqs. (33), (34), (39), (44), and (45) have been used. Note that repeated indices are summed over in Eqs. (46), (50), (51), (54), and (55). Evidently the integrand in Eq. (46) vanishes due to contraction of the completely antisymmetric tensor $\epsilon_{\alpha \beta \delta \sigma}$ with the symmetric tensor $c^{\alpha}(k) c^{\delta}(k)$, i.e.,

$$
\sum_{\alpha \delta} \epsilon_{\alpha \beta \delta \sigma} c^{\alpha}(k) c^{\delta}(k)=0
$$

Hence, we have

$$
G_{\mu \nu}(0,0)=0 .
$$

Next consider the first-order term of the power series (43),

$$
\begin{aligned}
& \left.\frac{\partial}{\partial p_{\lambda}} G_{\mu \nu}(p, q)\right|_{p, q=0} \\
& =\int_{k}\left\{\operatorname{tr}\left[\left.\gamma_{5} e_{0}(k) \partial_{p}^{\lambda} d_{1, \mu}(p, k)\right|_{p=k} e_{0}(k) d_{1, \nu}(k, k) e_{0}(k)\right]\right. \\
& \left.\quad+\operatorname{tr}\left[\gamma_{5} \partial_{\lambda} e_{0}(k) d_{1, \mu}(k, k) e_{0}(k) d_{1, \nu}(k, k) e_{0}(k)\right]\right\}
\end{aligned}
$$

From Eq. (36), it is easy to see that

$$
\left.\partial_{p}^{\lambda} d_{1, \mu}(p, k)\right|_{p=k}=\sum_{\sigma} \gamma^{\sigma} h_{\lambda \mu}^{\sigma}
$$

where each term has one factor of the $\gamma$ matrix, and the explicit expression of $h_{\lambda \mu}^{\sigma}$ is not required for our purposes. Then the first term and the second term of the integrand (48) both vanish,

$$
\begin{aligned}
& \operatorname{tr}\left[\gamma_{5} e_{0}(k) \gamma \cdot h_{\lambda \mu}(k) e_{0}(k) \partial_{\nu} d_{0}(k) e_{0}(k)\right] \\
& \quad=\operatorname{tr}(1) \epsilon_{\alpha \beta \delta \sigma} c^{\alpha}(k) c^{\delta}(k) b(k) h_{\lambda \mu}^{\beta}(k) \partial_{\nu} d_{0}^{\sigma}(k)=0
\end{aligned}
$$

and

$$
\begin{aligned}
\operatorname{tr}[ & \left.\gamma_{5} \partial_{\lambda} e_{0}(k) \partial_{\mu} d_{0}(k) e_{0}(k) \partial_{\nu} d_{0}(k) e_{0}\right] \\
= & \operatorname{tr}(1)\left(\epsilon_{\delta \alpha \beta \sigma}+\epsilon_{\delta \alpha \sigma \beta}\right) c^{\beta}(k) b(k) \partial_{\lambda} c^{\delta}(k) \\
& \times \partial_{\mu} d_{0}^{\alpha}(k) \partial_{\nu} d_{0}^{\sigma}(k) \\
& +\operatorname{tr}(1) \epsilon_{\alpha \beta \sigma \delta} c^{\beta}(k) c^{\delta}(k) \partial_{\lambda} b(k) \partial_{\mu} d_{0}^{\alpha}(k) \partial_{\nu} d_{0}^{\sigma}(k) \\
= & 0,
\end{aligned}
$$

where Eqs. (33), (34), (39), (49), (44), (45), and (47) have been used. Thus

$$
\left.\frac{\partial}{\partial p_{\lambda}} G_{\mu \nu}(p, q)\right|_{p, q=0}=0 .
$$

Similarly, we have

$$
\left.\frac{\partial}{\partial q_{\sigma}} G_{\mu \nu}(p, q)\right|_{p, q=0}=0 .
$$

Now consider the second order term of the power series (43),

$$
\begin{aligned}
\left.\frac{\partial}{\partial p_{\lambda}} \frac{\partial}{\partial q_{\sigma}} G_{\mu \nu}(p, q)\right|_{p, q=0}= & \int_{k}\left\{\operatorname{tr}\left[\left.\gamma_{5} \partial_{\lambda} e_{0}(k) d_{1, \mu}(k, k) e_{0}(k) \partial_{q}^{\sigma} d_{1, \nu}(k, q)\right|_{q=k} e_{0}(k)\right]\right. \\
& +\operatorname{tr}\left[\left.\gamma_{5} e_{0}(k) \partial_{p}^{\lambda} d_{1, \mu}(p, k)\right|_{p=k_{0}} e_{0}(k) d_{1, \nu}(k, k) \partial_{\sigma} e_{0}(k)\right] \\
& +\operatorname{tr}\left[\left.\left.\gamma_{5} e_{0}(k) \partial_{p}^{\lambda} d_{1, \mu}(p, k)\right|_{p=k_{0}} e_{0}(k) \partial_{q}^{\sigma} d_{1, \nu}(k, q)\right|_{q=k} e_{0}(k)\right] \\
& \left.+\operatorname{tr}\left[\gamma_{5} \partial_{\lambda} e_{0}(k) d_{1, \mu}(k, k) e_{0}(k) d_{1, \nu}(k, k) \partial_{\sigma} e_{0}(k)\right]\right\} .
\end{aligned}
$$


Evidently, the first three terms of the integrand are zero, by symmetry. Explicitly, the first term is equal to

$$
\begin{aligned}
\operatorname{tr}\left[\gamma_{5}\right. & \left.\partial_{\lambda} e_{0}(k) \partial_{\mu} d_{0}(k) e_{0}(k) \gamma \cdot h_{\sigma \nu}(k) e_{0}(k)\right] \\
= & \operatorname{tr}(1)\left(\epsilon_{\delta \alpha \beta \gamma}+\epsilon_{\delta \alpha \gamma \beta}\right) c^{\beta}(k) b(k) \partial_{\lambda} c^{\delta}(k) \partial_{\mu} d_{0}^{\alpha}(k) \\
& \times h_{\sigma \nu}^{\gamma}(k) \\
+ & \operatorname{tr}(\mathbb{1}) \epsilon_{\alpha \beta \gamma \delta} c^{\beta}(k) c^{\delta}(k) \partial_{\lambda} b(k) \partial_{\mu} d_{0}^{\alpha}(k) h_{\sigma \nu}^{\gamma}(k) \\
= & 0,
\end{aligned}
$$

the second term is also zero since it has the same form of the first, and the third term is

$$
\begin{aligned}
& \operatorname{tr}\left[\gamma_{5} e_{0}(k) \gamma \cdot h_{\lambda \mu}(k) e_{0}(k) \gamma \cdot h_{\sigma \nu}(k) e_{0}(k)\right] \\
& \quad=\operatorname{tr}(\mathbb{1}) \epsilon_{\alpha \beta \delta \gamma} c^{\alpha}(k) c^{\delta}(k) b(k) h_{\lambda \mu}^{\beta}(k) h_{\sigma \nu}^{\gamma}(k)=0,
\end{aligned}
$$

where Eqs. (33), (34), (39), (49), (44), (45), and (47) have been used. Thus, Eq. (53) becomes

$$
\begin{aligned}
& \left.\frac{\partial}{\partial p_{\lambda}} \frac{\partial}{\partial q_{\sigma}} G_{\mu \nu}(p, q)\right|_{p, q=0} \\
& \quad=\int_{k} \operatorname{tr}\left\{\partial_{\lambda}\left[\gamma_{5} e_{0}(k)\right] \partial_{\mu} d_{0}(k) e_{0}(k) \partial_{\nu} d_{0}(k) \partial_{\sigma} e_{0}(k)\right\} .
\end{aligned}
$$

From Eqs. (33) and (34), it is easy to see that $e_{0}(k)$ also satisfies the Ginsparg-Wilson relation

$$
\gamma_{5}+e_{0}^{-1}(k) \gamma_{5} e_{0}(k)=2 \gamma_{5} e_{0}(k)
$$

which, after differentiation with respect to $k_{\lambda}$, gives

$$
\left[\partial_{\lambda} e_{0}^{-1}(k)\right] \gamma_{5} e_{0}(k)+e_{0}^{-1}(k) \gamma_{5}\left[\partial_{\lambda} e_{0}(k)\right]=2 \partial_{\lambda}\left[\gamma_{5} e_{0}(k)\right] \text {. }
$$

Substituting Eq. (58) and $d_{0}(k)=e_{0}^{-1}(k)-1$ [from Eq. (18)] into the integrand of Eq. (56), then the integrand becomes

$$
\begin{aligned}
\frac{1}{2} \operatorname{tr} & \left\{\partial_{\lambda} e_{0}^{-1}(k) \gamma_{5} e_{0}(k) \partial_{\mu} e_{0}^{-1}(k) e_{0}(k) \partial_{\nu} e_{0}^{-1}(k) \partial_{\sigma} e_{0}(k)\right\} \\
& +\frac{1}{2} \operatorname{tr}\left\{e_{0}^{-1}(k) \gamma_{5} \partial_{\lambda} e_{0}(k) \partial_{\mu} e_{0}^{-1}(k) e_{0}(k) \partial_{\nu} e_{0}^{-1}(k)\right. \\
& \left.\times \partial_{\sigma} e_{0}(k)\right\}
\end{aligned}
$$

which can be further reduced to

$$
\begin{aligned}
& -\frac{1}{2} \operatorname{tr}\left\{\gamma_{5} \partial_{\mu} e_{0}(k) \partial_{\nu} e_{0}^{-1}(k) \partial_{\sigma} e_{0}(k) \partial_{\lambda} e_{0}^{-1}(k)\right\} \\
& +\frac{1}{2} \operatorname{tr}\left\{\gamma_{5} \partial_{\lambda} e_{0}(k) \partial_{\mu} e_{0}^{-1}(k) \partial_{\nu} e_{0}(k) \partial_{\sigma} e_{0}^{-1}(k)\right\},
\end{aligned}
$$

where the identities

$$
\begin{aligned}
e_{0}(k) \partial_{\mu} e_{0}^{-1}(k) e_{0}(k) & =-\partial_{\mu} e_{0}(k), \\
e_{0}^{-1}(k) \partial_{\mu} e_{0}(k) e_{0}^{-1}(k) & =-\partial_{\mu} e_{0}^{-1}(k),
\end{aligned}
$$

have been used. By symmetry, it is obvious that the contribution of the second expression of Eq. (59) is equal to that of the first one; thus Eq. (56) becomes

$$
\begin{aligned}
I_{\mu \nu \lambda \sigma} \equiv & \left.\frac{\partial}{\partial p_{\lambda}} \frac{\partial}{\partial q_{\sigma}} G_{\mu \nu}(p, q)\right|_{p, q=0} \\
= & \int \frac{d^{4} k}{(2 \pi)^{4}} \operatorname{tr}\left\{\gamma_{5} \partial_{\mu} e_{0}(k) \partial_{\nu} e_{0}^{-1}(k) \partial_{\lambda} e_{0}(k) \partial_{\sigma} e_{0}^{-1}(k)\right\} \\
= & \int \frac{d^{4} k}{(2 \pi)^{4}} \operatorname{tr}\left\{\gamma_{5} \partial_{\mu}\left(\frac{1}{1+d_{0}(k)}\right) \partial_{\nu} d_{0}(k)\right. \\
& \left.\times \partial_{\lambda}\left(\frac{1}{1+d_{0}(k)}\right) \partial_{\sigma} d_{0}(k)\right\},
\end{aligned}
$$

where the domain of integration is the four-torus, $\mathcal{T}_{4}=$ $\otimes_{i=1}^{4}[-\pi / a, \pi / a]$, in which the end points $( \pm \pi / a)$ in each direction are identified to be the same point. In the limit $a$ $\rightarrow 0, \mathcal{T}_{4} \rightarrow \otimes_{i=1}^{4}(-\infty, \infty)$, which is invariant under the transformation

$$
k_{\mu} \rightarrow-\frac{1}{r^{2} k_{\mu}}, \quad \mu=1, \ldots, 4,
$$

for any $r \neq 0$.

Now we change the variables according to relation (63); then Eq. (62) becomes

$$
\begin{aligned}
I_{\mu \nu \lambda \sigma}= & \int \frac{d^{4} k}{(2 \pi)^{4}} \operatorname{tr}\left\{\gamma_{5} \partial_{\mu}\left(\frac{1}{1+d_{0}(K)}\right) \partial_{\nu} d_{0}(K)\right. \\
& \left.\times \partial_{\lambda}\left(\frac{1}{1+d_{0}(K)}\right) \partial_{\sigma} d_{0}(K)\right\},
\end{aligned}
$$

where the arguments of $d_{0}$ are $K_{\mu}=-\left(r^{2} k_{\mu}\right)^{-1}$. Note that $d k_{\mu} \partial_{\mu}$ is invariant under the transformation (63).

Therefore, our ansatz of evaluating Eq. (64) in the continuum limit is to substitute $d_{0}(K)$ by $d_{0}^{-1}(k)$ in the integrand of Eq. (64), since $d_{0}(k) \rightarrow$ irak as $a \rightarrow 0$, and the trace with $\gamma_{5}$ picks out four distinct $\gamma$ matrices, one from each of the four factors with partial derivatives. Then Eq. (64) reads as

$$
\begin{aligned}
I_{\mu \nu \lambda \sigma}= & \int \frac{d^{4} k}{(2 \pi)^{4}} \operatorname{tr}\left\{\gamma_{5} \partial_{\mu}\left(\frac{d_{0}(k)}{1+d_{0}(k)}\right) \partial_{\nu} d_{0}^{-1}(k)\right. \\
& \left.\times \partial_{\lambda}\left(\frac{d_{0}(k)}{1+d_{0}(k)}\right) \partial_{\sigma} d_{0}^{-1}(k)\right\} \\
= & \int \frac{d^{4} k}{(2 \pi)^{4}} \operatorname{tr}\left\{\gamma_{5} \partial_{\mu} h(k) \partial_{\nu} h^{-1}(k) \partial_{\lambda} h(k) \partial_{\sigma} h^{-1}(k)\right\},
\end{aligned}
$$


where

$$
h(k)=\frac{d_{0}(k)}{1+d_{0}(k)} .
$$

The integral (65) can be evaluated by first removing an infinitesimal ball $B_{\epsilon}$ of radius $\epsilon$ from the origin $(k=0)$ of the four-torus $\mathcal{T}_{4}$, then performing the integration, and finally taking $\epsilon \rightarrow 0$, i.e.,

$$
\begin{aligned}
I_{\mu \nu \lambda \sigma}= & \frac{1}{16 \pi^{4}} \lim _{\epsilon \rightarrow 0} \int_{\mathcal{T}_{4} \backslash B_{\epsilon}} d^{4} k \\
& \times \operatorname{tr}\left\{\gamma_{5} \partial_{\mu} h(k) \partial_{\nu} h^{-1}(k) \partial_{\lambda} h(k) \partial_{\sigma} h^{-1}(k)\right\} \\
= & \frac{1}{16 \pi^{4}} \lim _{\epsilon \rightarrow 0} \int_{\mathcal{T}_{4} \backslash B_{\epsilon}} d^{4} k \\
& \times \partial_{\mu} \operatorname{tr}\left\{\gamma_{5} h(k) \partial_{\nu} h^{-1}(k) \partial_{\lambda} h(k) \partial_{\sigma} h^{-1}(k)\right\},
\end{aligned}
$$

where the $\partial_{\mu}$ operation in Eq. (67) produces Eq. (66), plus three terms that are symmetric in $\mu \nu, \mu \lambda$, and $\mu \sigma$, respectively; hence none one of these three terms contributes to the integral.

Then according to the Gauss theorem, the volume integral over $\mathcal{T}_{4} \backslash B_{\epsilon}$ can be expressed as a surface integral on the surface $\mathcal{S}_{\epsilon}$ of the ball $B_{\epsilon}$, provided that the integrand is continuous in $\mathcal{T}_{4} \backslash B_{\epsilon}$. The last condition is satisfied since $h(k)=r a D_{0}(k)$ is analytic, and $h(k) \neq 0$ (free of species doublings, for $0<c<2$ ) for any $k \in \mathcal{T}_{4} \backslash B_{\epsilon}$ [1]. Thus Eq. (67) becomes

$$
\begin{aligned}
I_{\mu \nu \lambda \sigma}= & \frac{1}{16 \pi^{4}} \lim _{\epsilon \rightarrow 0} \int_{S_{\epsilon}} d^{3} s n_{\mu} \\
& \times \operatorname{tr}\left\{\gamma_{5} h(k) \partial_{\nu} h^{-1}(k) \partial_{\lambda} h(k) \partial_{\sigma} h^{-1}(k)\right\},
\end{aligned}
$$

where $n_{\mu}$ is the $\mu$ th component of the outward normal vector on the surface $S_{\epsilon}$. Since $d_{0}(k) \rightarrow$ irak as $k \rightarrow 0$, we can set $d_{0}(k)=i r a k$ on the surface $S_{\epsilon}$ and obtain

$$
\begin{aligned}
I_{\mu \nu \lambda \sigma}= & \frac{1}{16 \pi^{4}} \lim _{\epsilon \rightarrow 0} \int_{S_{\epsilon}} d^{3} s n_{\mu} \\
& \times \operatorname{tr}\left\{\gamma_{5}\left(\frac{k}{1+r^{2} a^{2} k^{2}}\right) \frac{\gamma_{\nu}}{k^{2}}\left(\frac{\gamma_{\lambda}}{1+r^{2} a^{2} k^{2}}\right) \frac{\gamma_{\sigma}}{k^{2}}\right\}, \\
= & \frac{1}{16 \pi^{4}} \operatorname{tr}(1) \epsilon_{\mu \nu \lambda \sigma} \lim _{\epsilon \rightarrow 0} \int_{S_{\epsilon}} d^{3} s \frac{n_{\mu} k_{\mu}}{k^{4}\left(1+r^{2} a^{2} k^{2}\right)^{2}},
\end{aligned}
$$

where we have used Eqs. (44) and (45), and the property

$$
\int_{S_{\epsilon}} d^{3} s n_{\mu} k_{\nu} F\left(k^{2}\right)=\delta_{\mu \nu} \int_{S_{\epsilon}} d^{3} s n_{\mu} k_{\mu} F\left(k^{2}\right) .
$$

Finally, the result of the integral (69) is

$$
\begin{aligned}
I_{\mu \nu \lambda \sigma}= & -\frac{N_{f}}{4 \pi^{4}} \epsilon_{\mu \nu \lambda \sigma} \lim _{\epsilon \rightarrow 0} \frac{1}{\left(1+r^{2} a^{2} \epsilon^{2}\right)^{2}} \\
& \times \int_{0}^{2 \pi} d \phi \int_{0}^{\pi} d \theta_{2} \sin \theta_{2} \int_{0}^{\pi} d \theta_{1} \sin ^{2} \theta_{1} \cos ^{2} \theta_{1} \\
= & -\frac{N_{f}}{8 \pi^{2}} \epsilon_{\mu \nu \lambda \sigma},
\end{aligned}
$$

where $N_{f}$ denotes the number of fermion flavors. Note that Eq. (70) is independent of the parameter $r$ in $D$ [Eq. (1)]. From Eqs. (70) and (41), we obtain

$$
M(c)=\frac{N_{f}}{32 \pi^{2}}, \quad 0<c<2,
$$

and the axial anomaly (42) at order $g^{2}$,

$$
\begin{aligned}
\mathcal{A}_{2}(x)= & \frac{g^{2} N_{f}}{32 \pi^{2}} \sum_{\mu \nu \lambda \sigma} \epsilon_{\mu \nu \lambda \sigma} \\
& \times \operatorname{tr}\left\{\left(\partial_{\mu} A_{\nu}-\partial_{\nu} A_{\mu}\right)\left(\partial_{\lambda} A_{\sigma}-\partial_{\sigma} A_{\lambda}\right)(x)\right\},
\end{aligned}
$$

where

$$
A_{\mu}(x)=\int_{p} e^{i p \cdot x} \widetilde{A}_{\mu}(p)
$$

etc.

\section{B. The axial anomaly at order $g^{3}$}

Inserting the order $g^{3}$ terms of Eq. (17) into Eq. (15), we get $^{3}$

$$
\begin{aligned}
\mathcal{A}_{3}(x)= & g^{3} \int_{p} \int_{q} e^{i(p-q) \cdot x}\left\{J_{a}(p, q)+J_{b}(p, q)\right\} \\
& -g^{3} \int_{p} \int_{r} \int_{q} e^{i(p-q) \cdot x} J_{c}(p, r, q),
\end{aligned}
$$

where

$$
\begin{aligned}
J_{a}(p, q)= & \int_{k} \operatorname{tr}\left\{\gamma_{5} e_{0}(p) d_{1}(p, k) e_{0}(k) d_{2}(k, q) e_{0}(q)\right\}, \\
J_{b}(p, q)= & \int_{k} \operatorname{tr}\left\{\gamma_{5} e_{0}(p) d_{2}(p, k) e_{0}(k) d_{1}(k, q) e_{0}(q)\right\}, \\
J_{c}(p, r, q)= & \int_{k} \operatorname{tr}\left\{\gamma_{5} e_{0}(p) d_{1}(p, r) e_{0}(r) d_{1}(r, k) e_{0}(k)\right. \\
& \left.\times d_{1}(k, q) e_{0}(q)\right\},
\end{aligned}
$$

${ }^{3}$ The term $e_{0} d_{3} e_{0}$ has been dropped since $\operatorname{tr}\left(\gamma_{5} e_{0} d_{3} e_{0}\right)=0$. 
and $e_{0}$ and $d_{1}$ are given in Eqs. (33) and (35) respectively. It is easy to show that $J_{a}$ and $J_{b}$ vanish in the continuum limit, and only $J_{c}$ has a nonzero contribution to the axial anomaly at the order $g^{3}$. To proceed, we derive $d_{2}$ [Eq. (20)] by expanding $f$ and $t^{\mu}$ in power series of the gauge coupling [Eqs. (23) and (24)] similar to the procedure of deriving $d_{1}$ in Sec. II A. First, we solve for $f_{2}$ from the higher order equations (27), then together with the formulas of $t_{n}^{\mu}[\mathrm{Eq}$. (A17)] and $w_{n}[\mathrm{Eq},(\mathrm{A} 18)]$ derived in the Appendix, we obtain

$$
\begin{aligned}
d_{2}(p, k) & =g^{2} \sum_{\mu} \widetilde{A}_{\mu}^{(2)}(p-k) d_{2, \mu}(p, k) \\
& =g^{2} \sum_{\mu, \nu} \widetilde{A}_{\mu}^{(2)}(p-k) \gamma^{\nu} d_{2, \mu}^{\nu}(p, k),
\end{aligned}
$$

where $\widetilde{A}_{\mu}^{(2)}(p-k)$ is defined in Eq. (A9), and the explicit expression of $d_{2, \mu}^{\nu}(p, k)$ is not required for our subsequent calculations. In general, to any order of $g$, we have

$$
\begin{aligned}
d_{n}(p, k) & =g^{n} \sum_{\mu} \widetilde{A}_{\mu}^{(n)}(p-k) d_{n, \mu}(p, k) \\
& =g^{n} \sum_{\mu, \nu} \widetilde{A}_{\mu}^{(n)}(p-k) \gamma^{\nu} d_{n, \mu}^{\nu}(p, k),
\end{aligned}
$$

which can be used for higher-order calculations.

Using Eqs. (35) and (74), and changing variables $p \rightarrow p$ $+k$ and $q \rightarrow q+k$ in $J_{a}$ and $J_{b}$, while $p \rightarrow p+r+k, r \rightarrow r$ $+k$, and $q \rightarrow q+k$ in $J_{c}$, we can then rewrite Eq. (73) as

$$
\begin{aligned}
\mathcal{A}_{3}(x)= & g^{3} \int_{p} \int_{q} e^{i(p-q) \cdot x}\left\{I_{a}(p, q)+I_{b}(p, q)\right\} \\
& -g^{3} \int_{p} \int_{r} \int_{q} e^{i(p+r-q) \cdot x} I_{c}(p, r, q),
\end{aligned}
$$

where

$$
\begin{aligned}
I_{a}(p, q) & =\sum_{\mu, \nu} \operatorname{tr}\left[\widetilde{A}_{\mu}(p) \tilde{A}_{\nu}^{(2)}(-q)\right] G_{a}^{\mu \nu}(p, q), \\
I_{b}(p, q) & =\sum_{\mu, \nu} \operatorname{tr}\left[\widetilde{A}_{\mu}^{(2)}(p) \tilde{A}_{\nu}(-q)\right] G_{b}^{\mu \nu}(p, q), \\
I_{c}(p, r, q) & =\sum_{\mu, \nu, \lambda} \operatorname{tr}\left[\tilde{A}_{\mu}(p) \tilde{A}_{\nu}(r) \tilde{A}_{\lambda}(-q)\right] G_{c}^{\mu \nu \lambda}(p, r, q),
\end{aligned}
$$

and

$$
\begin{aligned}
G_{a}^{\mu \nu}(p, q)= & \int_{k} \operatorname{tr}\left[\gamma_{5} e_{0}(p+k) d_{1, \mu}(p+k, k) e_{0}(k)\right. \\
& \left.\times d_{2, \nu}(k, q+k) e_{0}(q+k)\right]
\end{aligned}
$$

$$
\begin{aligned}
G_{b}^{\mu \nu}(p, q)= & \int_{k} \operatorname{tr}\left[\gamma_{5} e_{0}(p+k) d_{2, \mu}(p+k, k) e_{0}(k)\right. \\
& \left.\times d_{1, \nu}(k, q+k) e_{0}(q+k)\right] \\
G_{c}^{\mu \nu \lambda}(p, r, q)= & \int_{k} \operatorname{tr}\left[\gamma_{5} e_{0}(p+r+k)\right. \\
& \times d_{1, \mu}(p+r+k, r+k) e_{0}(r+k) \\
& \times d_{1, \nu}(r+k, k) e_{0}(k) d_{1, \lambda}(k, q+k) \\
& \left.\times e_{0}(q+k)\right] .
\end{aligned}
$$

In the following, we show that $G_{a}$ and $G_{b}$ vanish in the continuum limit. First, we expand Eq. (80) in powers series of $p$ and $q$ :

$$
\begin{aligned}
G_{a}^{\mu \nu}(p, q)= & G_{a}^{\mu \nu}(0,0)+\left.\sum_{\sigma} p^{\sigma} \frac{\partial}{\partial p^{\sigma}} G_{a}^{\mu \nu}(p, 0)\right|_{p=0} \\
& +\left.\sum_{\sigma} q^{\sigma} \frac{\partial}{\partial q^{\sigma}} G_{a}^{\mu \nu}(0, q)\right|_{q=0}+O(a) ;
\end{aligned}
$$

where the zeroth-order and first-order terms can be easily shown to be zero, by symmetry. Explicitly,

$G_{a}^{\mu \nu}(0,0)=\int_{k} \operatorname{tr}\left[\gamma_{5} e_{0}(k) d_{1, \mu}(k, k) e_{0}(k) d_{2, \nu}(k, k) e_{0}(k)\right]=0$,

since the integrand vanishes identically,

$$
\begin{array}{r}
\operatorname{tr}\left[\gamma_{5} e_{0}(k) \partial_{\mu} e_{0}^{-1}(k) e_{0}(k) d_{2, \nu}(k, k) e_{0}(k)\right] \\
=-\operatorname{tr}\left[\gamma_{5} \partial_{\mu} e_{0}(k) d_{2, \nu}(k, k) e_{0}(k)\right]=0,
\end{array}
$$

where Eqs. (33), (39), (60), and (44) have been used.

To evaluate the first-order terms in Eq. (83), we observe that $^{4}$

$$
\begin{aligned}
& \left.\frac{\partial}{\partial p^{\sigma}} d_{1, \mu}(p+k, k)\right|_{p=0} \equiv \sum_{\lambda} \gamma^{\lambda} h_{\sigma \mu}^{\lambda}(k)=\gamma \cdot h_{\sigma \mu}(k), \\
& \left.\frac{\partial}{\partial q^{\sigma}} d_{2, \nu}(k, q+k)\right|_{q=0} \equiv \sum_{\lambda} \gamma^{\lambda} g_{\sigma \nu}^{\lambda}(k)=\gamma \cdot g_{\sigma \nu}(k),
\end{aligned}
$$

which follow from Eqs. (19), (20), (36), and (74), where the explicit expressions of $h_{\sigma \mu}^{\lambda}(k)$ and $g_{\sigma \nu}^{\lambda}(k)$ are not required for our subsequent calculations. Then

\footnotetext{
${ }^{4}$ Note that each term of $d_{1}$ [Eq. (19)] and $d_{2}$ [Eq. (20)] has only one factor of the $\gamma$ matrix.
} 


$$
\begin{aligned}
\frac{\partial}{\partial p^{\sigma}} G_{a}^{\mu \nu}(p, 0) & \left.\right|_{p=0} \\
= & \int_{k} \operatorname{tr}\left\{\gamma_{5}\left[\partial_{\sigma} e_{0}(k) \partial_{\mu} d_{0}(k)+e_{0}(k) \gamma \cdot h_{\sigma \mu}(k)\right]\right. \\
& \left.\times e_{0}(k) d_{2, \nu}(k, k) e_{0}(k)\right\}=0,
\end{aligned}
$$

since its integrand is equal to

$$
\begin{aligned}
\operatorname{tr}(1) & \boldsymbol{\epsilon}_{\alpha \beta \lambda \delta}\left\{\left[\partial_{\sigma} b(k) \partial_{\mu} d_{0}^{\alpha}(k)+b(k) h_{\sigma \mu}^{\alpha}(k)\right]\right. \\
& \left.\times c^{\beta}(k) c^{\delta}(k) d_{2, \nu}^{\lambda}(k)\right\}+\operatorname{tr}(\mathbb{1})\left(\epsilon_{\alpha \beta \lambda \delta}+\epsilon_{\alpha \beta \delta \lambda}\right) \\
& \times\left\{\left[\partial_{\sigma} c^{\alpha}(k) \partial_{\mu} d_{0}^{\beta}(k)+c^{\alpha}(k) h_{\sigma \mu}^{\beta}(k)\right] c^{\lambda}(k) d_{2, \nu}^{\delta}(k)\right\}=0,
\end{aligned}
$$

where Eqs. (33), (34), (84), (44), (45), and (47) have been used. Similarly, we have

$$
\begin{aligned}
\left.\frac{\partial}{\partial q^{\sigma}} G_{a}^{\mu \nu}(0, q)\right|_{q=0}= & \int_{k} \operatorname{tr}\left\{\gamma_{5} e_{0}(k) \partial_{\mu} d_{0}(k) e_{0}(k)\right. \\
& \times\left[\gamma \cdot g_{\sigma \nu}(k) e_{0}(k)\right. \\
& \left.\left.+d_{2, \nu}(k, k) \partial_{\sigma} e_{0}(k)\right]\right\}=0 .
\end{aligned}
$$

Therefore $G_{a}^{\mu \nu}(p, q)=O(a) \rightarrow 0$ in the limit $a \rightarrow 0$. By the same token, $G_{b}^{\mu \nu}(p, q)=0$ in the continuum limit.

Next we expand $G_{c}$ in power series of $p, q$ and $r$,

$$
\begin{aligned}
G_{c}^{\mu \nu \lambda}(p, r, q)= & G_{c}^{\mu \nu \lambda}(0,0,0)+\left.\sum_{\sigma} p^{\sigma} \frac{\partial}{\partial p^{\sigma}} G_{c}^{\mu \nu \lambda}(p, 0,0)\right|_{p=0} \\
& +\left.\sum_{\sigma} r^{\sigma} \frac{\partial}{\partial r^{\sigma}} G_{c}^{\mu \nu \lambda}(0, r, 0)\right|_{r=0} \\
& +\left.\sum_{\sigma} q^{\sigma} \frac{\partial}{\partial q^{\sigma}} G_{c}^{\mu \nu \lambda}(0,0, q)\right|_{q=0}+O(a) .
\end{aligned}
$$

It is easy to see that the zeroth-order term vanishes,

$$
\begin{aligned}
G_{c}^{\mu \nu \lambda}(0,0,0)= & \int_{k} \operatorname{tr}\left[\gamma_{5} e_{0}(k) d_{1, \mu}(k, k) e_{0}(k) d_{1, \nu}(k, k) e_{0}(k)\right. \\
& \left.\times d_{1, \lambda}(k, k) e_{0}(k)\right]=0
\end{aligned}
$$

since its integrand vanishes,

$$
\begin{aligned}
\operatorname{tr}\left[\gamma_{5} e_{0}(k) d_{1, \mu}(k, k) e_{0}(k) d_{1, \nu}(k, k) e_{0}(k) d_{1, \lambda}(k, k) e_{0}(k)\right] \\
=\operatorname{tr}\left[\gamma_{5} e_{0}(k) \partial_{\mu} e_{0}^{-1}(k) e_{0}(k) \partial_{\nu} e_{0}^{-1}(k) e_{0}(k)\right. \\
\left.\quad \times \partial_{\lambda} e_{0}^{-1}(k) e_{0}(k)\right] \\
=\operatorname{tr}\left[\gamma_{5} \partial_{\mu} e_{0}(k) \partial_{\nu} e_{0}^{-1}(k) \partial_{\lambda} e_{0}(k)\right]=0
\end{aligned}
$$

where Eqs. (39), (60), and (44) have been used.

Next we evaluate the first-order terms

$$
\begin{aligned}
\left.\frac{\partial}{\partial p^{\sigma}} G_{c}^{\mu \nu \lambda}(p, 0,0)\right|_{p=0} & \\
= & -\int_{k} \operatorname{tr}\left\{\gamma_{5}\left[\partial_{\sigma} e_{0}(k) \partial_{\mu} e_{0}^{-1}(k)+e_{0}(k) \gamma \cdot h_{\sigma \mu}(k)\right]\right. \\
& \left.\times e_{0}(k) \partial_{\nu} e_{0}^{-1}(k) \partial_{\lambda} e_{0}(k)\right\} \\
= & -\int_{k} \operatorname{tr}\left\{\gamma_{5} \partial_{\sigma} e_{0}(k) \partial_{\mu} e_{0}^{-1}(k) e_{0}(k) \partial_{\nu} e_{0}^{-1}(k) \partial_{\lambda} e_{0}(k)\right\},
\end{aligned}
$$

where Eqs. (39), (84) and (60) have been used, and the last equality is due to the vanishing of the second term [containing the factor $\left.\gamma \cdot h_{\sigma \mu}(k)\right]$ in the integrand,

$$
\begin{aligned}
\operatorname{tr}\left\{\gamma_{5} e_{0}(k) \gamma \cdot h_{\sigma \mu}(k) e_{0}(k) \partial_{\nu} e_{0}^{-1}(k) \partial_{\lambda} e_{0}(k)\right\} \\
=\operatorname{tr}(1) \epsilon_{\alpha \beta \gamma \delta} \partial_{\lambda} b(k) c^{\alpha}(k) c^{\gamma} h_{\sigma \mu}^{\beta}(k) \partial_{\nu} d_{0}^{\delta}(k) \\
\quad+\operatorname{tr}(\mathbb{1})\left(\epsilon_{\alpha \beta \gamma \delta}+\epsilon_{\beta \alpha \gamma \delta}\right) b(k) c^{\alpha}(k) \partial_{\lambda} c^{\delta}(k) h_{\sigma \mu}^{\beta}(k) \\
\quad \times \partial_{\nu} d_{0}^{\gamma}(k)=0 .
\end{aligned}
$$

Comparing Eqs. (88) with (56), one immediately obtains ${ }^{5}$

$$
\left.\frac{\partial}{\partial p^{\sigma}} G_{c}^{\mu \nu \lambda}(p, 0,0)\right|_{p=0}=I_{\mu \nu \lambda \sigma}=-\frac{N_{f}}{8 \pi^{2}} \epsilon_{\mu \nu \lambda \sigma},
$$

which has been evaluated in Eq. (70).

Similarly, we obtain

$$
\left.\frac{\partial}{\partial q^{\sigma}} G_{c}^{\mu \nu \lambda}(0,0, q)\right|_{q=0}=-I_{\mu \nu \lambda \sigma}=\frac{N_{f}}{8 \pi^{2}} \epsilon_{\mu \nu \lambda \sigma} .
$$

Next we evaluate

$$
\begin{aligned}
&\left.\frac{\partial}{\partial r^{\sigma}} G_{c}^{\mu \nu \lambda}(0, r, 0)\right|_{r=0} \\
&=\int_{k}\left(\operatorname { t r } \left[\gamma_{5} \partial_{\sigma} e_{0}(k) \partial_{\mu} e_{0}^{-1}(k) e_{0}(k) \partial_{\nu} e_{0}^{-1}(k) e_{0}(k)\right.\right. \\
&\left.\quad \times \partial_{\lambda} e_{0}^{-1}(k) e_{0}(k)\right] \\
& \quad+\operatorname{tr}\left[\gamma_{5} e_{0}(k) \partial_{\mu} e_{0}^{-1}(k) \partial_{\sigma} e_{0}(k) \partial_{\nu} e_{0}^{-1}(k)\right. \\
&\left.\quad \times e_{0}(k) \partial_{\lambda} e_{0}^{-1}(k) e_{0}(k)\right] \\
& \quad+\operatorname{tr}\left[\gamma_{5} e_{0}(k) \partial_{\sigma} \partial_{\mu} d_{0}(k) e_{0}(k) \partial_{\nu} e_{0}^{-1}(k) e_{0}(k)\right. \\
&\left.\quad \times \partial_{\lambda} e_{0}^{-1}(k) e_{0}(k)\right]+\operatorname{tr}\left\{\gamma_{5} e_{0}(k) \partial_{\mu} e_{0}^{-1}(k) e_{0}(k)\right. \\
&\left.\left.\quad \times\left[\gamma \cdot h_{\sigma \nu}(k)\right] e_{0}(k) \partial_{\lambda} e_{0}^{-1}(k) e_{0}(k)\right\}\right) \\
&= 0,
\end{aligned}
$$

\footnotetext{
${ }^{5}$ Note that $\partial_{\mu} e_{0}^{-1}(k)=\partial_{\mu} d_{0}(k)$, from Eq. (33).
} 
where in the integrand of Eq. (91), the first two terms cancel each other, and the last two terms vanish respectively, after the (by now familiar) manipulations using Eq. (33), (39), (60), (61), (44), (45), and (47).

Substituting Eqs. (87), (89), (90), and (92) into Eq. (86), we obtain

$$
G_{c}^{\mu \nu \lambda}(p, r, q)=-\frac{N_{f}}{8 \pi^{2}} \sum_{\sigma}\left(p_{\sigma}-q_{\sigma}\right) \epsilon_{\mu \nu \lambda \sigma}+O(a) .
$$

Therefore, in the continuum limit, the axial anomaly (76) at $O\left(g^{3}\right)$ is

$$
\begin{aligned}
\mathcal{A}_{3}(x)= & \frac{g^{3} N_{f}}{8 \pi^{2}} \sum_{\mu \nu \lambda \sigma} \epsilon_{\mu \nu \lambda \sigma} \int_{p} \int_{r} \int_{q} e^{i(p+r-q) \cdot x}\left(p_{\sigma}-q_{\sigma}\right) \\
& \times \operatorname{tr}\left[\widetilde{A}_{\mu}(p) \widetilde{A}_{\nu}(r) \widetilde{A}_{\lambda}(-q)\right] \\
= & -i \frac{g^{3} N_{f}}{32 \pi^{2}} \sum_{\mu \nu \lambda \sigma} \epsilon_{\mu \nu \lambda \sigma} \operatorname{tr}\left\{\left[\partial_{\sigma} A_{\mu}(x)-\partial_{\mu} A_{\sigma}(x)\right]\right. \\
& \times\left[A_{\nu}(x), A_{\lambda}(x)\right]+\left[A_{\mu}(x), A_{\nu}(x)\right] \\
& \left.\times\left[\partial_{\sigma} A_{\lambda}(x)-\partial_{\lambda} A_{\sigma}(x)\right]\right\},
\end{aligned}
$$

where

$$
A_{\mu}(x)=\int_{p} e^{i p \cdot x} \widetilde{A}_{\mu}(p)
$$

etc.

\section{The axial anomaly up to order $g^{4}$}

Inserting the order $g^{4}$ terms of Eq. (17) into Eq. (15), we have $^{6}$

$$
\begin{aligned}
\mathcal{A}_{4}(x)= & g^{4} \int_{p} \int_{q} e^{i(p-q) \cdot x}\left\{J_{1}(p, q)+J_{2}(p, q)+J_{3}(p, q)\right\} \\
& -g^{4} \int_{p} \int_{r} \int_{q} e^{i(p-q) \cdot x}\left\{J_{4}(p, r, q)+J_{5}(p, r, q)\right. \\
& \left.+J_{6}(p, r, q)\right\}+g^{4} \int_{p} \int_{r} \int_{s} \int_{q} e^{i(p-q) \cdot x} J_{7}(p, r, s, q),
\end{aligned}
$$

where

$$
\begin{aligned}
& J_{1}(p, q)=\int_{k} \operatorname{tr}\left[\gamma_{5} e_{0}(p) d_{1}(p, k) e_{0}(k) d_{3}(k, q) e_{0}(q)\right], \\
& J_{2}(p, q)=\int_{k} \operatorname{tr}\left[\gamma_{5} e_{0}(p) d_{2}(p, k) e_{0}(k) d_{2}(k, q) e_{0}(q)\right],
\end{aligned}
$$

\footnotetext{
${ }^{6}$ The term $e_{0} d_{4} e_{0}$ has been dropped since $\operatorname{tr}\left(\gamma_{5} e_{0} d_{4} e_{0}\right)=0$.
}

$$
\begin{aligned}
J_{3}(p, q)= & \int_{k} \operatorname{tr}\left[\gamma_{5} e_{0}(p) d_{3}(p, k) e_{0}(k) d_{1}(k, q) e_{0}(q)\right], \\
J_{4}(p, r, q)= & \int_{k} \operatorname{tr}\left[\gamma_{5} e_{0}(p) d_{1}(p, r) e_{0}(r) d_{1}(r, k) e_{0}(k)\right. \\
& \left.\times d_{2}(k, q) e_{0}(q)\right], \\
J_{5}(p, r, q)= & \int_{k} \operatorname{tr}\left[\gamma_{5} e_{0}(p) d_{1}(p, r) e_{0}(r) d_{2}(r, k) e_{0}(k)\right. \\
& \left.\times d_{1}(k, q) e_{0}(q)\right], \\
J_{6}(p, r, q)= & \int_{k} \operatorname{tr}\left[\gamma_{5} e_{0}(p) d_{2}(p, r) e_{0}(r) d_{1}(r, k) e_{0}(k)\right. \\
& \left.\times d_{1}(k, q) e_{0}(q)\right], \\
J_{7}(p, r, s, q)= & \int_{k} \operatorname{tr}\left[\gamma_{5} e_{0}(p) d_{1}(p, r) e_{0}(r) d_{1}(r, s) e_{0}(s)\right. \\
& \left.\times d_{1}(s, k) e_{0}(k) d_{1}(k, q) e_{0}(q)\right] .
\end{aligned}
$$

Now we change the variables as follows:

(i) $p \rightarrow p+k$ and $q \rightarrow q+k$ in $J_{1}, J_{2}$, and $J_{3}$;

(ii) $p \rightarrow p+r+k, r \rightarrow r+k$, and $q \rightarrow q+k$ in $J_{4}, J_{5}$, and $J_{6}$;

(iii) $p \rightarrow p+r+s+k, r \rightarrow r+s+k, s \rightarrow s+k$, and $q \rightarrow q$ $+k$ in $J_{7}$. Then Eq. (95) becomes

$$
\begin{aligned}
\mathcal{A}_{4}(x)= & g^{4} \int_{p} \int_{q} e^{i(p-q) \cdot x}\left\{I_{1}(p, q)+I_{2}(p, q)+I_{3}(p, q)\right\} \\
& -g^{4} \int_{p} \int_{r} \int_{q} e^{i(p+r-q) \cdot x}\left\{I_{4}(p, r, q)+I_{5}(p, r, q)\right. \\
& \left.+I_{6}(p, r, q)\right\} \\
& +g^{4} \int_{p} \int_{r} \int_{s} \int_{q} e^{i(p+r+s-q) \cdot x} I_{7}(p, r, s, q),
\end{aligned}
$$

where

$$
I_{1}(p, q)=\sum_{\mu, \nu} \operatorname{tr}\left[\widetilde{A}_{\mu}(p) \widetilde{A}_{\nu}^{(3)}(-q)\right] G_{1}^{\mu \nu}(p, q)
$$$$
I_{2}(p, q)=\sum_{\mu, \nu} \operatorname{tr}\left[\widetilde{A}_{\mu}^{(2)}(p) \widetilde{A}_{\nu}^{(2)}(-q)\right] G_{2}^{\mu \nu}(p, q)
$$

$$
I_{3}(p, q)=\sum_{\mu, \nu} \operatorname{tr}\left[\widetilde{A}_{\mu}^{(3)}(p) \widetilde{A}_{\nu}(-q)\right] G_{3}^{\mu \nu}(p, q)
$$$$
I_{4}(p, r, q)=\sum_{\mu, \nu, \lambda} \operatorname{tr}\left[\widetilde{A}_{\mu}(p) \widetilde{A}_{\nu}(r) \tilde{A}_{\lambda}^{(2)}(-q)\right] G_{4}^{\mu \nu \lambda}(p, r, q)
$$$$
I_{5}(p, r, q)=\sum_{\mu, \nu, \lambda} \operatorname{tr}\left[\widetilde{A}_{\mu}(p) \widetilde{A}_{\nu}^{(2)}(r) \widetilde{A}_{\lambda}(-q)\right] G_{5}^{\mu \nu \lambda}(p, r, q)
$$ 


$$
\begin{aligned}
I_{6}(p, r, q)= & \sum_{\mu, \nu, \lambda} \operatorname{tr}\left[\tilde{A}_{\mu}^{(2)}(p) \tilde{A}_{\nu}(r) \tilde{A}_{\lambda}(-q)\right] G_{6}^{\mu \nu \lambda}(p, r, q) \\
I_{7}(p, r, s, q)= & \sum_{\mu, \nu, \lambda, \sigma} \operatorname{tr}\left[\tilde{A}_{\mu}(p) \tilde{A}_{\nu}(r) \tilde{A}_{\lambda}(s) \tilde{A}_{\sigma}(-q)\right] \\
& \times G_{7}^{\mu \nu \lambda \sigma}(p, r, s, q)
\end{aligned}
$$

and

$$
\begin{aligned}
& G_{1}^{\mu \nu}(p, q)=\int_{k} \operatorname{tr}\left[\gamma_{5} e_{0}(p+k) d_{1, \mu}(p+k, k) e_{0}(k)\right. \\
& \left.\times d_{3, \nu}(k, q+k) e_{0}(q+k)\right] \\
& G_{2}^{\mu \nu}(p, q)=\int_{k} \operatorname{tr}\left[\gamma_{5} e_{0}(p+k) d_{2, \mu}(p+k, k) e_{0}(k)\right. \\
& \left.\times d_{2, \nu}(k, q+k) e_{0}(q+k)\right] \\
& G_{3}^{\mu \nu}(p, q)=\int_{k} \operatorname{tr}\left[\gamma_{5} e_{0}(p+k) d_{3, \mu}(p+k, k) e_{0}(k)\right. \\
& \left.\times d_{1, \nu}(k, q+k) e_{0}(q+k)\right], \\
& G_{4}^{\mu \nu \lambda}(p, r, q)=\int_{k} \operatorname{tr}\left[\gamma_{5} e_{0}(p+r+k) d_{1, \mu}(p+r+k, r+k)\right. \\
& \times e_{0}(r+k) d_{1, \nu}(r+k, k) e_{0}(k) \\
& \left.\times d_{2, \lambda}(k, q+k) e_{0}(q+k)\right] \text {, } \\
& G_{5}^{\mu \nu \lambda}(p, r, q)=\int_{k} \operatorname{tr}\left[\gamma_{5} e_{0}(p+r+k) d_{1, \mu}(p+r+k, r+k)\right. \\
& \times e_{0}(r+k) d_{2, \nu}(r+k, k) e_{0}(k) \\
& \left.\times d_{1, \lambda}(k, q+k) e_{0}(q+k)\right] \text {, } \\
& G_{6}^{\mu \nu \lambda}(p, r, q)=\int_{k} \operatorname{tr}\left[\gamma_{5} e_{0}(p+r+k) d_{2, \mu}(p+r+k, r+k)\right. \\
& \times e_{0}(r+k) d_{1, \nu}(r+k, k) e_{0}(k) \\
& \left.\times d_{1, \lambda}(k, q+k) e_{0}(q+k)\right] \text {, } \\
& G_{7}^{\mu \nu \lambda \sigma}(p, r, s, q)=\int_{k} \operatorname{tr}\left[\gamma_{5} e_{0}(p+r+s+k)\right. \\
& \times d_{1, \mu}(p+r+s+k, r+s+k) \\
& \times e_{0}(r+s+k) d_{1, \nu}(r+s+k, s+k) \\
& \times e_{0}(s+k) d_{1, \lambda}(s+k, k) e_{0}(k) \\
& \left.\times d_{1, \sigma}(k, q+k) e_{0}(q+k)\right],
\end{aligned}
$$

where Eqs. (35), (74), and (75) (for $n=3$ ) have been used, and $\widetilde{A}_{\mu}^{(n)}(p)$, etc., are defined in Eq. (A9).
In the following, we show that all $G$ ' $s$ vanish in the continuum limit except $G_{7}$, by expanding each one of them in power series of $p, q, r$ and $s$. First consider

$$
G_{1}^{\mu \nu}(p, q)=G_{1}^{\mu \nu}(0,0)+O(a)
$$

where

$$
G_{1}^{\mu \nu}(0,0)=\int_{k} \operatorname{tr}\left[\gamma_{5} e_{0}(k) d_{1, \mu}(k, k) e_{0}(k) d_{3, \nu}(k, k) e_{0}(k)\right]=0
$$

Since its integrand vanishes,

$$
\begin{aligned}
\operatorname{tr} & {\left[\gamma_{5} e_{0}(k) d_{1, \mu}(k, k) e_{0}(k) d_{3, \nu}(k, k) e_{0}(k)\right] } \\
& =\operatorname{tr}(1) \epsilon_{\alpha \beta \gamma \delta} b(k) c^{\alpha}(k) c^{\gamma}(k) \partial_{\mu} d_{0}^{\beta}(k) d_{3, \nu}^{\delta}(k)=0,
\end{aligned}
$$

where Eqs. (33), (39), (75), (44), (45), and (47) have been used. Similarly, we obtain

$$
G_{2}^{\mu \nu}(0,0)=G_{3}^{\mu \nu}(0,0)=0 .
$$

Next consider

$$
G_{4}^{\mu \nu \lambda}(p, r, q)=G_{4}^{\mu \nu \lambda}(0,0,0)+O(a)
$$

where

$$
\begin{aligned}
G_{4}^{\mu \nu \lambda}(0,0,0)= & \int_{k} \operatorname{tr}\left[\gamma_{5} e_{0}(k) d_{1, \mu}(k, k) e_{0}(k) d_{1, \nu}(k, k)\right. \\
& \left.\times e_{0}(k) d_{2, \lambda}(k, k) e_{0}(k)\right]=0,
\end{aligned}
$$

Since its integrand vanishes,

$$
\begin{aligned}
\operatorname{tr}\left[\gamma_{5} e_{0}(k) \partial_{\mu} e_{0}^{-1}(k) e_{0}(k) \partial_{\nu} d_{0}(k) e_{0}(k) d_{2, \lambda}(k, k) e_{0}(k)\right] \\
=-\operatorname{tr}\left[\gamma_{5} \partial_{\mu} e_{0}(k) \partial_{\nu} d_{0}(k) e_{0}(k) d_{2, \lambda}(k, k) e_{0}(k)\right] \\
=-\operatorname{tr}(\mathbb{1})\left\{\epsilon_{\alpha \beta \gamma \delta} \partial_{\mu} b(k) c^{\beta}(k) c^{\delta}(k) \partial_{\nu} d_{0}^{\alpha}(k) d_{2, \lambda}^{\gamma}(k)\right\} \\
\quad+\operatorname{tr}(\mathbb{1})\left\{\left(\epsilon_{\alpha \beta \gamma \delta}+\epsilon_{\alpha \beta \delta \gamma}\right) b(k) c^{\gamma}(k) \partial_{\mu} c^{\alpha}(k) \partial_{\nu} d_{0}^{\beta}(k)\right. \\
\left.\quad \times d_{2, \lambda}^{\delta}(k)\right\}=0,
\end{aligned}
$$

where Eqs. (33), (39), (60), (74), (44), (45), and (47) have been used. By the same token, we have

$$
G_{5}^{\mu \nu \lambda}(0,0,0)=G_{6}^{\mu \nu \lambda}(0,0,0)=0 .
$$

Finally, we consider

$$
G_{7}^{\mu \nu \lambda \sigma}(p, r, s, q)=G_{7}^{\mu \nu \lambda \sigma}(0,0,0,0)+O(a),
$$

where 


$$
\begin{aligned}
G_{7}^{\mu \nu \lambda \sigma}(0,0,0,0)= & \int_{k} \operatorname{tr}\left[\gamma_{5} e_{0}(k) d_{1, \mu}(k, k) e_{0}(k) d_{1, \nu}(k, k)\right. \\
& \left.\times e_{0}(k) d_{1, \lambda}(k, k) e_{0}(k) d_{1, \sigma}(k, k) e_{0}(k)\right] \\
= & \int_{k} \operatorname{tr}\left[\gamma_{5} e_{0}(k) \partial_{\mu} e_{0}^{-1}(k) e_{0}(k) \partial_{\nu} e_{0}^{-1}(k)\right. \\
& \left.\times e_{0}(k) \partial_{\lambda} e_{0}^{-1}(k) e_{0}(k) \partial_{\sigma} e_{0}^{-1}(k) e_{0}(k)\right] \\
= & \int_{k} \operatorname{tr}\left[\gamma_{5} \partial_{\mu} e_{0}(k) \partial_{\nu} e_{0}^{-1}(k) e_{0}(k) \partial_{\lambda} e_{0}^{-1}(k)\right. \\
& \left.\times \partial_{\sigma} e_{0}(k)\right] .
\end{aligned}
$$

Here we have used Eqs. (39) and (60) to simplify the integrand. Comparing Eqs. (103) with (56), one immediately obtains

$$
G_{7}^{\mu \nu \lambda \sigma}(0,0,0,0)=I_{\mu \nu \lambda \sigma}=-\frac{N_{f}}{8 \pi^{2}} \epsilon_{\mu \nu \lambda \sigma},
$$

which has been evaluated in Eq. (70).

Thus, in the continuum limit, only $G_{7}$ has a nonzero contribution to the axial anomaly. Substituting Eq. (102) into Eq. (96), we obtain the axial anomaly at the order $g^{4}$,

$$
\begin{aligned}
\mathcal{A}_{4}(x)= & -\frac{g^{4} N_{f}}{8 \pi^{2}} \sum_{\mu \nu \lambda \sigma} \epsilon_{\mu \nu \lambda \sigma} \int_{p} \int_{r} \int_{s} \int_{q} e^{i(p+r+s-q) \cdot x} \\
& \times \widetilde{A}_{\mu}(p) \widetilde{A}_{\nu}(r) \widetilde{A}_{\lambda}(s) \widetilde{A}_{\sigma}(-q) \\
= & -\frac{g^{4} N_{f}}{8 \pi^{2}} \sum_{\mu \nu \lambda \sigma} \epsilon_{\mu \nu \lambda \sigma} \operatorname{tr}\left\{A_{\mu}(x) A_{\nu}(x) A_{\lambda}(x) A_{\sigma}(x)\right\} \\
= & -\frac{g^{4} N_{f}}{32 \pi^{2}} \sum_{\mu \nu \lambda \sigma} \epsilon_{\mu \nu \lambda \sigma} \operatorname{tr}\left\{\left[A_{\mu}(x), A_{\nu}(x)\right]\right. \\
& \left.\times\left[A_{\lambda}(x), A_{\sigma}(x)\right]\right\} .
\end{aligned}
$$

Finally, adding Eqs. (72), (94), and (105) gives the axial anomaly (15) in the continuum limit,

$$
\begin{aligned}
\mathcal{A}(x) & =\operatorname{tr}\left[\gamma_{5}(1-\operatorname{ar} D)(x, x)\right] \\
& =\frac{g^{2} N_{f}}{32 \pi^{2}} \sum_{\mu \nu \lambda \sigma} \epsilon_{\mu \nu \lambda \sigma} \operatorname{tr}\left\{F_{\mu \nu}(x) F_{\lambda \sigma}(x)\right\},
\end{aligned}
$$

where

$$
F_{\mu \nu}=\partial_{\mu} A_{\nu}-\partial_{\nu} A_{\mu}+i g\left[A_{\mu}, A_{\nu}\right]
$$

Note that the axial anomaly (106) is invariant for any $r>0$, since the anomaly coefficient (70) is independent of $r$. This completes our perturbation calculation of the axial anomaly of the Ginsparg-Wilson lattice Dirac operator proposed in Ref. [1].
TABLE I. The ratio of the integral $I_{1234}$ [Eq. (62)] to $1 / 8 \pi^{2}$, for lattices of sizes $16^{4}, 32^{4}, 64^{4}, 128^{4}, 256^{4}$, as well as for $r$ $=0.5,1 \cdot 0,2.0,4.0$, respectively. The lattice spacing $a$ is set to one, and the parameter $c$ [Eqs. (3) and (8)] is fixed at 1.0.

\begin{tabular}{lcccc}
\hline \hline$L$ & \multicolumn{4}{c}{$r$} \\
\hline \multirow{4}{*}{16} & 0.5 & 1.0 & 2.0 & 4.0 \\
32 & 0.9204 & 0.9800 & 0.9848 & 0.7617 \\
64 & 0.9810 & 0.9952 & 0.9988 & 0.9907 \\
128 & 0.9953 & 0.9988 & 0.9997 & 0.9999 \\
256 & 0.9988 & 0.9997 & 0.9999 & 1.0000 \\
\hline \hline
\end{tabular}

\section{CONCLUDING REMARKS}

Several remarks are as follows. It is obvious that Eq. (106) also holds for other $T_{\mu}$ [Eq. (3)] such that $D$ is doubler-free, is exponentially local, and has correct continuum behavior. In particular, Eq. (106) holds for

$$
f=\left(\frac{2 c}{\sqrt{t^{2}+w^{2}}+w}\right)^{\alpha}, \quad \alpha \geqslant \frac{1}{2}, \quad 0<c<2,
$$

as proposed in Ref. [1].

It is instructive to examine how well the continuum axial anomaly can be recovered on a finite lattice, by evaluating the integral $I_{1234}[\mathrm{Eq} .(62)]$ as a numerical sum over the discrete momenta on a finite lattice. In Table I, the ratios of $I_{1234}$ to $1 / 8 \pi^{2}$ are listed for several lattice sizes $\left(L^{4}\right)$, as well as for a range of $r$, respectively. Here the lattice spacing $a$ is set to one, and the value of $c$ [see Eqs. (3) and (8)] is fixed at 1.0. Evidently, the integral $I_{1234}$ tends to the continuum value $1 / 8 \pi^{2}$ as $L \rightarrow \infty$, independent of the parameter $r$. This also provides a verification of our ansatz to substitute $d_{0}(K)$ by $d_{0}^{-1}(k)$ in evaluating $I_{\mu \nu \lambda \sigma}$ [Eq. (64)] in the continuum limit.

Next we repeat the same calculations of $I_{1234}$ for another $f$, namely, replacing Eq. (3) by

$$
f=\frac{2 c}{\sqrt{t^{2}+w^{2}}+w} .
$$

The results are listed in Table II, which show that the integral $I_{1234}$ (as a function of lattice size $L^{4}$ ) approaches the con-

TABLE II. The ratio of the integral $I_{1234}$ [Eq. (62)] to $1 / 8 \pi^{2}$, for $f=2 c /\left(\sqrt{t^{2}+w^{2}}+w\right)$. Other parameters are the same as those in Table I.

\begin{tabular}{lcccc}
\hline \hline$L$ & \multicolumn{5}{c}{$r$} \\
\hline & 0.5 & 1.0 & 2.0 & 4.0 \\
16 & 1.0000 & 1.0000 & 0.9911 & 0.7565 \\
32 & 1.0000 & 1.0000 & 1.0000 & 0.9914 \\
64 & 1.0000 & 1.0000 & 1.0000 & 1.0000 \\
128 & 1.0000 & 1.0000 & 1.0000 & 1.0000 \\
256 & 1.0000 & 1.0000 & 1.0000 & 1.0000 \\
\hline \hline
\end{tabular}


tinuum value $1 / 8 \pi^{2}$ much faster than that in Table I. This indicates that Eq. (109) may be a better choice than Eq. (3), especially for small lattices.

In general, $T_{\mu}$ [Eq. (2)] may not be in the form $T_{\mu}$ $=f t_{\mu} f$; then our present perturbation calculation may not go through without modifications. We refer to our former derivation [6] for the general case, though some of our intermediate steps need further clarifications. If one assumes that the coefficient of the axial anomaly, $g^{2} / 32 \pi^{2}$, is the same for $U(1)$ and $S U(n)$ background gauge fields, then it can also be determined [8] by imposing a gauge configuration with constant field tensors to Lüscher's formula [7] for the axial anomaly of Ginsparg-Wilson lattice Dirac fermions in a $U(1)$ background gauge field, provided that $D$ is topologically proper. However, for a topologically trivial GinspargWilson lattice Dirac operator such as Eq. (1), it seems to be necessary to perform an explicit calculation in order to determine its axial anomaly. In passing, we also refer to other axial anomaly calculations [9-12] for the overlap Dirac operator $[13,14]$, as well as that in the original Ginsparg-Wilson paper [2]. So far, a nonperturbative analytic calculation of the axial anomaly for any Ginsparg-Wilson lattice Dirac operator, on a finite lattice, is still lacking.

In summary, we have shown that the lattice Dirac operator (1) reproduces the continuum axial anomaly for smooth gauge configurations, even though it does not possess any topological zero modes in topologically nontrivial gauge backgrounds. If one insists that the topologically zero modes of a lattice Dirac operator are crucial for lattice QCD to reproduce low energy hadron phenomenology, then one should ensure that a Ginsparg-Wilson lattice Dirac operator is indeed topologically proper, before it could be employed for lattice QCD computations. However, so far, there does not seem to be compelling experimental evidence that these topological zero modes are physically relevant, unlike the axial anomaly in the trivial gauge sector, which accounts for the decay rate of the neutral pion. So there might be a very slight possibility that lattice QCD with topologically trivial quarks could reproduce low energy hadron phenomenology. These issues seem to deserve further study.

\section{ACKNOWLEDGMENTS}

This work was supported in part by the National Science Council, Republic of China, under Grant Nos. NSC89-2112M002-079 and NSC90-2112-M002-021. We would like to thank David Adams for discussions.

\section{APPENDIX: BASIC FORMULAS IN WEAK COUPLING PERTURBATION THEORY}

In this appendix, we set up our notation by deriving some basic formulas in weak coupling perturbation theory. These formulas are used in our anomaly calculation in Sec. II.

Consider the forward and backward difference operators

$$
\nabla_{\mu}^{+}(x, y) \equiv U_{\mu}(x) \delta_{x+a \hat{\mu}, y}-\delta_{x, y},
$$

$$
\nabla_{\mu}^{-}(x, y) \equiv \delta_{x, y}-U_{\mu}^{\dagger}(x-a \hat{\mu}) \delta_{x-a \hat{\mu}, y},
$$

where the link variables [for the $S U(n)$ gauge group] are defined as

$$
\begin{aligned}
U_{\mu}(x) & =\exp \left[\operatorname{iagA_{\mu }}\left(x+\frac{a}{2} \hat{\mu}\right)\right], \\
U_{\mu}^{\dagger}(x-a \hat{\mu}) & =\exp \left[-i a g A_{\mu}\left(x-\frac{a}{2} \hat{\mu}\right)\right] .
\end{aligned}
$$

Expanding $\nabla^{ \pm}$in power series of the gauge coupling $g$,

$$
\nabla_{\mu}^{ \pm}=\left(\nabla_{\mu}^{ \pm}\right)_{0}+\left(\nabla_{\mu}^{ \pm}\right)_{1}+\left(\nabla_{\mu}^{ \pm}\right)_{2}+\cdots,
$$

and performing the Fourier transform

$$
\sum_{x, y} e^{-i(p \cdot x-q \cdot y)}\left(\nabla_{\mu}^{+}\right)_{n}(x, y) \equiv\left(\nabla_{\mu}^{+}\right)_{n}(p, q),
$$

we obtain

$$
\left(\nabla_{\mu}^{+}\right)_{0}(p, q)=\left(e^{i p_{\mu} a}-1\right) \delta^{4}(p-q) \equiv\left(\nabla_{\mu}^{+}\right)_{0}(p) \delta^{4}(p-q)
$$

and

$$
\begin{aligned}
\left(\nabla_{\mu}^{+}\right)_{n}(p, q)= & \sum_{x, y} e^{-i(p \cdot x-q \cdot y)} \frac{1}{n !}(i a g)^{n} \\
& \times\left[A_{\mu}\left(x+\frac{a}{2} \hat{\mu}\right)\right]^{n} \delta_{x+a \hat{\mu}, y} \\
= & \sum_{x} e^{-i(p-q) \cdot(x+a \hat{\mu} / 2)} \frac{1}{n !}(i a g)^{n} \\
& \times\left[A_{\mu}\left(x+\frac{a}{2} \hat{\mu}\right)\right]^{n} e^{i\left(p_{\mu}+q_{\mu}\right) a / 2} \\
= & \frac{(i a g)^{n}}{n !} \widetilde{A}_{\mu}^{(n)}(p-q) e^{i\left(p_{\mu}+q_{\mu}\right) a / 2} \\
= & \frac{g^{n}}{n !} \widetilde{A}_{\mu}^{(n)}(p-q) \partial_{\mu}^{n}\left(\nabla_{\mu}^{+}\right)_{0}\left(\frac{p+q}{2}\right),
\end{aligned}
$$

where

$$
\widetilde{A}_{\mu}^{(n)}(p-q)=\sum_{x} e^{-i(p-q) \cdot(x+a \hat{\mu} / 2)}\left[A_{\mu}\left(x+\frac{a}{2} \hat{\mu}\right)\right]^{n},
$$

$$
\left(\nabla_{\mu}^{+}\right)_{0}\left(\frac{p+q}{2}\right)=e^{i\left(p_{\mu}+q_{\mu}\right) a / 2}-1 .
$$

Similarly, we have

$$
\left(\nabla_{\mu}^{-}\right)_{0}(p, q)=\left(1-e^{-i p_{\mu} a}\right) \delta^{4}(p-q) \equiv\left(\nabla_{\mu}^{-}\right)_{0}(p) \delta^{4}(p-q),
$$




$$
\left(\nabla_{\mu}^{-}\right)_{n}(p, q)=\frac{g^{n}}{n !} \widetilde{A}_{\mu}^{(n)}(p-q) \partial_{\mu}^{n}\left(\nabla_{\mu}^{-}\right)_{0}\left(\frac{p+q}{2}\right)
$$

(A12)

Then we obtain the weak coupling perturbation series of $t^{\mu}$ [Eq. (4)] and $w$ [Eq. (8)] in the momentum space:

$$
\begin{aligned}
t^{\mu}(p, q) & =\frac{1}{2}\left[\nabla_{\mu}^{+}(p, q)+\nabla_{\mu}^{-}(p, q)\right] \\
& =t_{0}^{\mu}(p) \delta^{4}(p-q)+t_{1}^{\mu}(p, q)+t_{2}^{\mu}(p, q)+\cdots, \\
w(p, q) & =c-\frac{1}{2} \sum_{\mu}\left[\nabla_{\mu}^{+}(p, q)+\nabla_{\mu}^{-}(p, q)\right] \\
& =w_{0}(p) \delta^{4}(p-q)+w_{1}(p, q)+w_{2}(p, q)+\cdots,
\end{aligned}
$$

[1] T.W. Chiu, Phys. Lett. B 521, 429 (2001).

[2] P.H. Ginsparg and K.G. Wilson, Phys. Rev. D 25, 2649 (1982).

[3] M. Lüscher, Phys. Lett. B 428, 342 (1998).

[4] S. Weinberg, The Quantum Theory of Fields. Vol. 2: Modern Applications (Cambridge University Press, Cambridge, England, 1996).

[5] W.A. Bardeen, Phys. Rev. 184, 1848 (1969).

[6] T.W. Chiu and T.H. Hsieh, hep-lat/9901011. where

$$
t_{0}^{\mu}(p)=i \sin \left(p_{\mu} a\right)
$$

$w_{0}(p)=c-\sum_{\mu}\left[1-\cos \left(p_{\mu} a\right)\right]$

$t_{n}^{\mu}(p, q)=\frac{g^{n}}{n !} \widetilde{A}_{\mu}^{(n)}(p-q) \partial_{\mu}^{n} t_{0}^{\mu}\left(\frac{p+q}{2}\right)$,

$$
w_{n}(p, q)=\frac{g^{n}}{n !} \sum_{\mu} \widetilde{A}_{\mu}^{(n)}(p-q) \partial_{\mu}^{n} w_{0}\left(\frac{p+q}{2}\right) .
$$

[7] M. Lüscher, Nucl. Phys. B538, 515 (1999).

[8] T.W. Chiu, Phys. Lett. B 445, 371 (1999).

[9] Y. Kikukawa and A. Yamada, Phys. Lett. B 448, 265 (1999).

[10] D.H. Adams, hep-lat/9812003.

[11] K. Fujikawa, Nucl. Phys. B546, 480 (1999).

[12] H. Suzuki, Prog. Theor. Phys. 102, 141 (1999).

[13] H. Neuberger, Phys. Lett. B 417, 141 (1998).

[14] R. Narayanan and H. Neuberger, Nucl. Phys. B443, 305 (1995) 